\title{
First Total Synthesis of Artekeiskeanol A, C and Altissimacoumarin D
}

\author{
Anil Talakokkula \\ Karunakar Baikadi \\ A. Venkat Narsaiah* \\ Organic Synthesis Laboratory, Fluoro-Agrochemicals Department, \\ CSIR-Indian Institute of Chemical Technology, Hyderabad, 500007, \\ Telangana, India \\ vnakkirala2001@yahoo.com
}

IICT Communication No: IICT/Pubs./2019/066

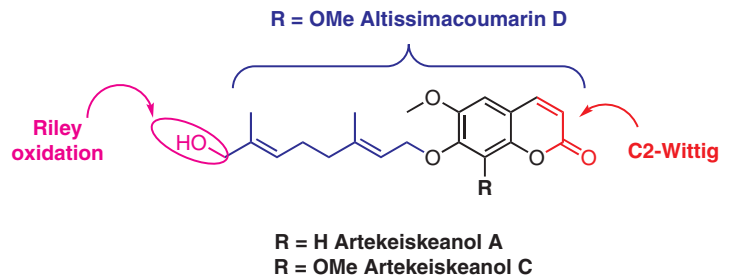

$\mathbf{R}=$ OMe Artekeiskeanol C
Received: 21.02.2019

Accepted after revision: 17.03.2019

Published online: 02.04 .2019

DOI: 10.1055/s-0037-1610873; Art ID: so-2019-d0007-op

License terms: cc)

Abstract The total syntheses of artekeiskeanol A and C, and altissimacoumarin $D$ have been achieved. The syntheses commenced from commercially available starting materials, 2,4-dihydroxybenzaldehyde and geraniol. The key steps involve Wittig and Riley oxidation reactions.

Keywords geronyl, scopoletin, isofraxidin, altissimacoumarin, artekeiskeanol

Coumarins are privileged core units in many bioactive molecules and they are responsible for scavenging of reactive oxygen species (ROS). ${ }^{1}$ Scopoletin [6-methoxy-7hydroxycoumarin], is obtained from the stem extracts of Erycibe obtusifolia Benth, a traditional medicine of China with multiple bioactivities. ${ }^{2}$ Isofraxidin [7-hydroxy-6,8dimethoxy-2H-chromen-2-one], isolated mainly from Acansopanax senticosus, exhibits antitumor activity on human hepatoma cell lines HuH-7 and HepG2. ${ }^{3}$ Altissimacou- marin D [(E)-7-[(3,7-dimethylocta-2,6-dien-1-yl)oxy]-6,8dimethoxy-2H-chromen-2-one], isolated from the Chinese medicinal plant, Alianthus altissima, exhibits significant Stir1 activation and its structure was confirmed by $\mathrm{Oh}$ et al. ${ }^{4}$

Artekeiskeanols A-D (Figure 1) were isolated by Kwak et al. ${ }^{5}$ from Artemisia keiskeana Miq (Compositae), which is used as a Korean traditional medicine for amenorrhea, gynaecopathy, bruising and rheumatic diseases. Their structures were reported by Schmitz et al. ${ }^{6}$ and their antigenstimulant RBL-2H3 mast cell activation through inhibition of Akt, JNK and P38 cells was reported by Honga et al. ${ }^{7}$ As part of our research program on the synthesis of biologically active natural and synthetic molecules, ${ }^{8}$ we herein report the first total syntheses of artekeiskeanol A and C, and also the structurally related altissimacoumarin D.

As shown in the retrosynthetic analysis (Scheme 1), the natural coumarins scopoletin (6) and isofraxidin (10), were identified as key intermediates for the sequential synthesis of artekeiskeanol A (1), C (2) and altissimacoumarin D (3). Artekeiskeanol $A$ and $C$ could be obtained from compound

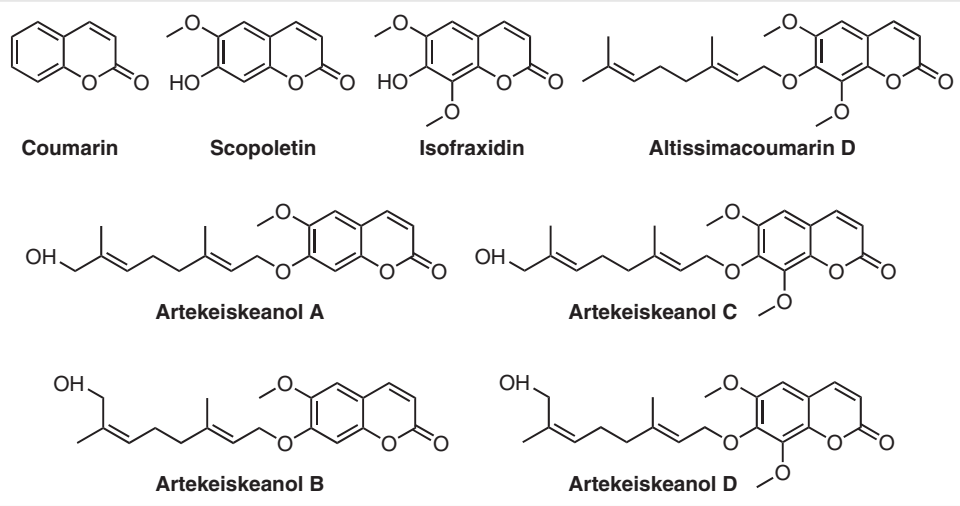

Figure 1 
Scheme 1

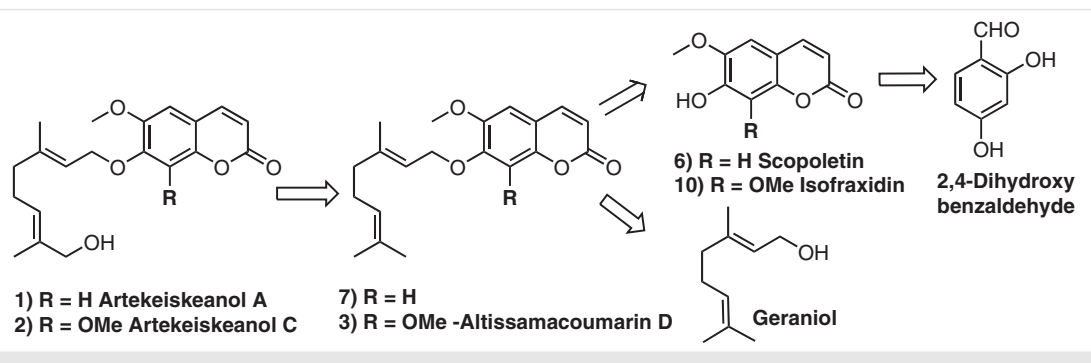

(7) and compound (3), respectively, via Riley oxidation. Intermediates (3) and (7) could be synthesized by reaction between geranyl bromide (11) with isofraxidin (10) or scopoletin (6). Isofraxidin and scopoletin may be synthesized from commercially available 2,4-dihydroxybenzaldehyde (Scheme 1).

Regioselective bromination ${ }^{9}$ of 2,4-dihydroxybenzaldehyde was carried out using bromine in an equimolar ratio with the substrate in methanol at room temperature to afford 5-bromo-2,4-dihydroxybenzaldehyde (4) in excellent yields. The monobromo compound 4 was then subjected to nucleophilic substitution with NaOMe in the presence of $\mathrm{CuCl}$ at reflux in DMF to furnish, 2,4-dihydroxy-5-methoxybenzaldehyde (5) in good yield. ${ }^{10}$ The resultant product $\mathbf{5}$ was subjected to Wittig olefination with ethyl (triphenylphosporanylidene) acetate, in $\mathrm{N}, \mathrm{N}$-diethylaniline at reflux to give the intermediate 7-hydroxy-6-methoxy-2Hchromen-2-one (6) (scopotletin) in 70\% yield. ${ }^{11}$

The intermediate $\mathbf{6}$ and geranyl bromide $(\mathbf{1 1})^{12}$ were reacted in the presence of $\mathrm{K}_{2} \mathrm{CO}_{3}$ in DMF at room temperature to produce $(E)-7-[(3,7-d i m e t h y l o c t a-2,6-d i e n-1-y l) o x y]-6-$ methoxy-2H-chromen-2-one $(7)^{13}$ in $82 \%$ yield, which, on oxidation with $\mathrm{SeO}_{2}$ and TBHP in $\mathrm{CH}_{2} \mathrm{Cl}_{2}$ at $0{ }^{\circ} \mathrm{C}$, afforded 7$\{[(2 E, 6 E)-8$-hydroxy-3,7-dimethylocta-2,6-dien-1-yl]oxy $\}$ 6 -methoxy-2H-chromen-2-one $(\mathbf{1})^{14}$ in $60 \%$ yield (Scheme 2 ), corresponding to artekeiskeanol A with spectroscopic data that was consistent with those reported (Table 1$){ }^{6}$
The synthesis of artekeiskeanol C (2) also started from 2,4-dihydroxybenzaldehyde, this time with an excess of bromine in methanol at room temperature to give, 3,5-dibromo-2,4-dihydroxybenzaldehyde (8) in quantitative yield. The dibromo compound $\mathbf{8}$ was subjected to nucleophilic substitution with $\mathrm{NaOMe}$ in the presence $\mathrm{CuCl}$ in $\mathrm{DMF}$ at reflux for $8 \mathrm{~h}$ to furnish, 2,4-dihydroxy-3,5-dimethoxybenzaldehyde (9) in 75\% yield. Compound $\mathbf{9}$ was subjected to Wittig olefination with ethyl (triphenylphosporanylidene) acetate, in $N, N$-diethylaniline at reflux to afford, 7-hydroxy-6,8-dimethoxy-2H-chromen-2-one (10) in $72 \%$ yield, corresponding to isofraxidin.

Isofraxidin, on reaction with geranyl bromide and $\mathrm{K}_{2} \mathrm{CO}_{3}$ in DMF at room temperature, afforded (E)-7-[(3,7-dimethylocta-2,6-dien-1-yl)oxy]-6,8-dimethoxy-2H-chromen-2one (3) in $80 \%$ yield, which corresponds to the natural product altissimacoumarin D, with spectroscopic data consistent with those reported. ${ }^{13 a}$ Again, we adopted the Riley protocol for oxidation of compound 3, with $\mathrm{SeO}_{2}$ and TBHP in $\mathrm{CH}_{2} \mathrm{Cl}_{2}$ at $0{ }^{\circ} \mathrm{C}$ to afford, $7-\{[(2 E, 6 E)-8$-hydroxy-3,7-dimethylocta-2,6-dien-1-yl]oxy\}-6,8-dimethoxy-2H-

chromen-2-one (2), in good yield (Scheme 3 ), to furnish artekeiskeanol $\mathrm{C}$. The spectroscopic data of the synthetic material was consistent with those reported for the natural product (Table 2$)^{6}$

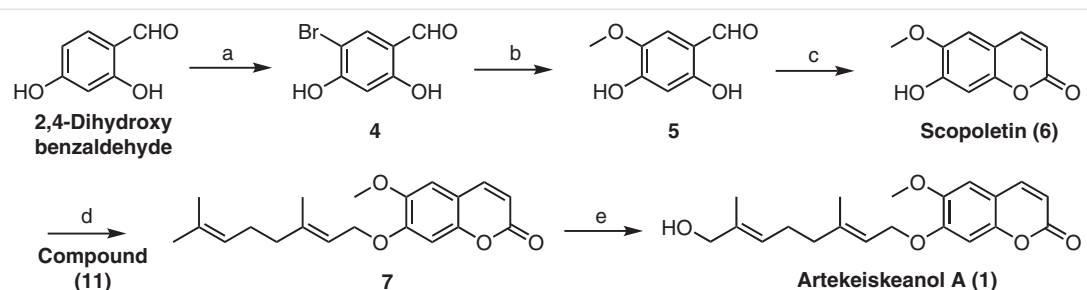

Scheme 2 Reagents and conditions: (a) Bromine, EtOH, r.t, 1 h, 80\%; (b) NaOMe, CuCl, DMF, reflux, 8 h, 75\%; (c) $\mathrm{C}_{2}$-Ylide, $N$, $N$-diethylaniline, reflux, 12 h, 70\%; (d) Geranyl bromide (11), $\mathrm{K}_{2} \mathrm{CO}_{3}, \mathrm{KI}$ (cat), DMF, r.t, 2 h, $82 \%$; (e) $\mathrm{SeO}_{2}, \mathrm{TBHP} \mathrm{CH}_{2} \mathrm{Cl}_{2}$ (2:1), $0{ }^{\circ} \mathrm{C}$ to r.t, 4 h, $60 \%$. 


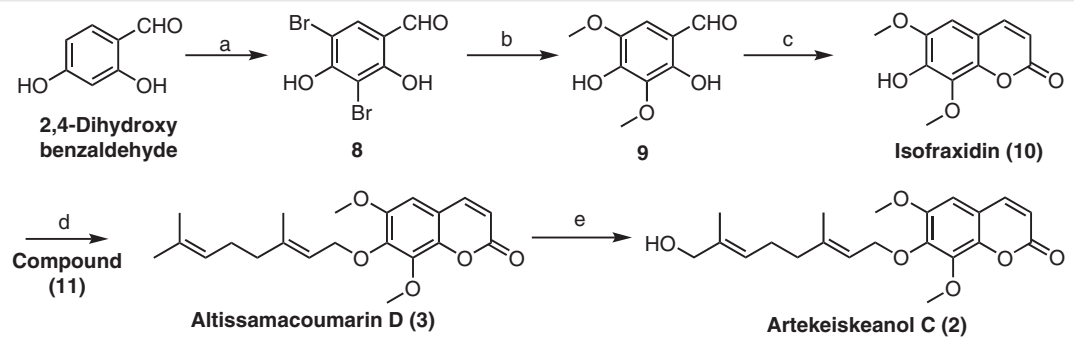

Scheme 3 Reagents and conditions: (a) $\mathrm{Br}_{2}$, EtOH, r.t, 1 h, 98\%; (b) $\mathrm{NaOMe}, \mathrm{CuCl}, \mathrm{DMF}$, reflux $8 \mathrm{~h}, 75 \%$; (c) C2-Ylide, $N, \mathrm{~N}$-diethylaniline, reflux, $12 \mathrm{~h}, 72 \%$; (d) Geranyl bromide (11), $\mathrm{K}_{2} \mathrm{CO}_{3}$, $\mathrm{KI}$ (cat), DMF, r.t, $2 \mathrm{~h}, 80 \%$; (e) $\mathrm{SeO}_{2}, \mathrm{TBHP} \mathrm{CH}_{2} \mathrm{Cl}_{2}(2: 1), 0{ }^{\circ} \mathrm{C}$ to r.t., 4 h, $63 \%$.

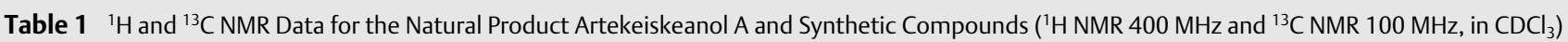

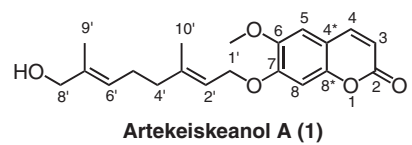

\begin{tabular}{|c|c|c|c|c|}
\hline \multirow[t]{2}{*}{${ }^{1} \mathrm{H} /{ }^{13} \mathrm{C}$ Position } & \multicolumn{2}{|c|}{ Artekeiskeanol A, Natural product } & \multicolumn{2}{|l|}{ Synthetic product } \\
\hline & ${ }^{1} \mathrm{H}$ NMR $\delta(\mathrm{ppm})(\mathrm{J}, \mathrm{Hz})$ & ${ }^{13} \mathrm{C}$ NMR (ppm) & ${ }^{1} \mathrm{H}$ NMR $\delta(\mathrm{ppm})(\mathrm{J}, \mathrm{Hz})$ & ${ }^{13} \mathrm{C}$ NMR (ppm) \\
\hline 2 & & 161.5 & & 161.5 \\
\hline 3 & $6.24(\mathrm{~d}, 9.4)$ & 113.3 & $6.28(\mathrm{~d}, 9.4)$ & 113.3 \\
\hline 4 & $7.59(d, 9.4)$ & 143.3 & $7.61(\mathrm{~d}, 9.4)$ & 143.3 \\
\hline $4^{*}$ & & 111.3 & & 111.3 \\
\hline 5 & $6.82(s)$ & 108.1 & $6.85(\mathrm{~s})$ & 108.0 \\
\hline 6 & & 146.6 & & 146.6 \\
\hline 7 & & 152.1 & & 152.1 \\
\hline 8 & $6.79(s)$ & 101.2 & $6.83(s)$ & 101.2 \\
\hline $8^{*}$ & & 149.9 & & 149.9 \\
\hline $1^{\prime}$ & $4.66(d, 6.3)$ & 66.2 & $4.69(\mathrm{~d}, 6.4)$ & 66.3 \\
\hline $2^{\prime}$ & $5.45(\mathrm{tdd}, 6.5,2.4,1.2)$ & 118.8 & $5.48(\mathrm{tdd}, 6.4,2.3,1.2)$ & 118.8 \\
\hline $3^{\prime}$ & & 141.5 & & 141.5 \\
\hline $4^{\prime}$ & $2.08(t, 7.5)$ & 39.0 & $2.10(\mathrm{~m})$ & 39.0 \\
\hline $5^{\prime}$ & $2.16(\mathrm{q}, 7.2)$ & 25.5 & $2.20(\mathrm{~m})$ & 25.5 \\
\hline $6^{\prime}$ & $5.34(\mathrm{tdd}, 6.9,2.6,1.3)$ & 125.0 & 5.37 (tdd, 6.9, 2.6, 1.4) & 125.0 \\
\hline $7^{\prime}$ & & 135.4 & & 135.4 \\
\hline $8^{\prime}$ & $3.94(s)$ & 68.7 & $3.97(s)$ & 68.8 \\
\hline $9^{\prime}$ & $1.63(\mathrm{~s})$ & 13.7 & $1.66(\mathrm{~s})$ & 13.7 \\
\hline $10^{\prime}$ & $1.74(\mathrm{~s})$ & 16.7 & $1.78(\mathrm{~s})$ & 16.8 \\
\hline $\mathrm{OCH}_{3}-6$ & $3.87(\mathrm{~s})$ & 56.3 & $3.91(\mathrm{~s})$ & 56.3 \\
\hline
\end{tabular}

In summary, we have executed the first total synthesis of artekeiskeanol $\mathrm{A}$ and $\mathrm{C}$ in five steps with overall yields of $21 \%$ and $27 \%$, respectively. The key reactions involved in this synthetic approach are a regioselective bromination reaction, Wittig olefination, followed by cyclization and Riley oxidation.
All air- and moisture-sensitive reactions were carried out under a nitrogen or argon atmosphere. Oven-dried glass apparatus was used to perform all reactions. Freshly distilled anhydrous solvents were used for air- and moisture-sensitive reactions. Commercially available reagents were used as such. Purification of compounds was carried out by column chromatography using silica gel (60-120 mesh). ${ }^{1} \mathrm{H}$ and ${ }^{13} \mathrm{C}$ NMR spectra were recorded in $\mathrm{CDCl}_{3}$ with 400 and $500 \mathrm{MHz}$ spectrometers, respectively, using TMS as internal standard. IR spec- 
Table $2{ }^{1} \mathrm{H}$ and ${ }^{13} \mathrm{C}$ NMR Data for the Natural Product Artekeiskeanol $\mathrm{C}$ and Synthetic Compounds $\left({ }^{1} \mathrm{H} \mathrm{NMR} 400 \mathrm{MHz}\right.$ and ${ }^{13} \mathrm{C} \mathrm{NMR}^{100 ~ M H z}$, in CDCl $)$

\begin{tabular}{|c|c|c|c|c|}
\hline \multicolumn{5}{|c|}{ Artekeiskeanol C (2) } \\
\hline \multirow[t]{2}{*}{${ }^{1} \mathrm{H} /{ }^{13} \mathrm{C}$ Position } & \multicolumn{2}{|c|}{ Artekeiskeanol C, Natural product } & \multicolumn{2}{|l|}{ Synthetic product } \\
\hline & ${ }^{1} \mathrm{H}$ NMR $\delta(\mathrm{ppm})(\mathrm{V}, \mathrm{Hz})$ & ${ }^{13} \mathrm{C}$ NMR (ppm) & ${ }^{1} \mathrm{H}$ NMR $\delta(\mathrm{ppm})(\mathrm{J}, \mathrm{Hz})$ & ${ }^{13} \mathrm{C}$ NMR (ppm) \\
\hline 2 & & 160.6 & & 160.5 \\
\hline 3 & $6.30(d, 9.5)$ & 115.1 & $6.34(d, 9.4)$ & 115.1 \\
\hline 4 & $7.58(\mathrm{~d}, 9.5)$ & 143.5 & $7.61(d, 9.5)$ & 143.4 \\
\hline $4^{*}$ & & 114.4 & & 114.4 \\
\hline 5 & $6.63(s)$ & 103.5 & $6.66(s)$ & 103.6 \\
\hline 6 & & 150.6 & & 150.6 \\
\hline 7 & & 144.7 & & 144.8 \\
\hline 8 & & 141.6 & & 141.6 \\
\hline $8^{*}$ & & 142.9 & & 142.9 \\
\hline $1^{\prime}$ & $4.63(\mathrm{~d}, 7.5)$ & 70.1 & $4.67(\mathrm{~d}, 7.2)$ & 70.2 \\
\hline $2^{\prime}$ & 5.52 (tdd, $6.4,2.3,1.3)$ & 119.8 & $5.56(\mathrm{tdd}, 7.2,2.3,1.2)$ & 119.9 \\
\hline $3^{\prime}$ & & 142.1 & & 142.1 \\
\hline $4^{\prime}$ & $2.04(t, 7.5)$ & 39.1 & $2.07(\mathrm{~m})$ & 39.1 \\
\hline $5^{\prime}$ & $2.11(\mathrm{q}, 7.0)$ & 25.7 & $2.17(\mathrm{~m})$ & 25.7 \\
\hline $6^{\prime}$ & $5.32(t, 7.0)$ & 125.2 & $5.37(t, 6.6)$ & 125.3 \\
\hline $7^{\prime}$ & & 135.1 & & 135.2 \\
\hline $8^{\prime}$ & $3.94(\mathrm{~s})$ & 68.7 & $3.98(\mathrm{~s})$ & 68.8 \\
\hline $9^{\prime}$ & $1.61(\mathrm{~s})$ & 13.6 & $1.66(\mathrm{~s})$ & 13.7 \\
\hline $10^{\prime}$ & $1.66(\mathrm{~s})$ & 16.3 & $1.70(\mathrm{~s})$ & 16.3 \\
\hline $\mathrm{OCH}_{3}-6$ & $3.85(\mathrm{~s})$ & 56.2 & $3.89(\mathrm{~s})$ & 56.3 \\
\hline $\mathrm{OCH}_{3}-8$ & $3.99(\mathrm{~s})$ & 61.7 & $4.03(\mathrm{~s})$ & 61.7 \\
\hline
\end{tabular}

tra were recorded with a Perkin-Elmer FT-RT 240-c Spectrophotometer using $\mathrm{KBr} /$ Thin Film optics. Mass spectra were recorded with a Finnigan MAT 1020 mass spectrometer operating at $70 \mathrm{eV}$. High-resolution mass spectra (HRMS) [ESI+] were obtained using either a TOF or a double focusing spectrometer.

\section{5-Bromo-2,4-dihydroxybenzaldehyde (4)}

To a stirred solution of 2,4-dihydroxybenzaldehyde ( $0.5 \mathrm{~g}, 3.62 \mathrm{mmol})$ in $\mathrm{EtOH}(10 \mathrm{~mL})$ was added dropwise bromine $(0.1 \mathrm{~mL}, 3.26 \mathrm{mmol})$ at r.t. and the mixture was stirred for $1 \mathrm{~h}$. After the completion of the reaction (monitored by TLC), the reaction was quenched with saturated aqueous sodium thiosulfate, the mixture was extracted with EtOAc $(2 \times 20 \mathrm{~mL})$, and the combined organic layers were washed with brine, dried over $\mathrm{Na}_{2} \mathrm{SO}_{4}$, and filtered. The solvent was evaporated under reduced pressure to give 4 .

Yield: $0.63 \mathrm{~g}(80 \%)$; off-white solid; $\mathrm{mp} 147-150^{\circ} \mathrm{C}$.

IR (neat): 3360, 2920, 1715, 1465, 780, $575 \mathrm{~cm}^{-1}$.

${ }^{1} \mathrm{H}$ NMR $\left(400 \mathrm{MHz}, \mathrm{CDCl}_{3}\right): \delta=11.24(\mathrm{~s}, 1 \mathrm{H}), 9.69(\mathrm{~s}, 1 \mathrm{H}), 7.65(\mathrm{~s}$, $1 \mathrm{H}), 6.62$ (s, $1 \mathrm{H}), 6.17$ (brs, $1 \mathrm{H})$.
${ }^{13} \mathrm{C}$ NMR $\left(100 \mathrm{MHz}, \mathrm{CDCl}_{3}\right): \delta=194.2,160.3,159.5,134.3,115.5$, 108.3, 98.7.

HRMS: $m / z$ [M - H] $]^{+}$calcd for $\mathrm{C}_{7} \mathrm{H}_{4} \mathrm{O}_{3} \mathrm{Br}$ : 214.9333; found: 214.9338 .

\section{2,4-Dihydroxy-5-methoxybenzaldehyde (5)}

To a stirred solution of sodium methoxide ( $1.2 \mathrm{~g}, 23.3 \mathrm{mmol})$ in DMF was added bromo compound $\mathbf{4}(0.5 \mathrm{~g}, 2.3 \mathrm{mmol})$ followed by $\mathrm{CuCl}(23$ $\mathrm{mg}, 0.23 \mathrm{mmol})$. The reaction mixture was heated to reflux for $8 \mathrm{~h}$ with vigorous stirring. After the completion of the reaction (monitored by TLC), solvent was removed under vacuum, cold water was added, and the mixture stirred for 20 min, neutralized with $\mathrm{HCl}$ then stirred for another $20 \mathrm{~min}$ and extracted with $\mathrm{CHCl}_{3}(2 \times 10 \mathrm{~mL})$. The separated organic layers were washed with brine, dried with $\mathrm{Na}_{2} \mathrm{SO}_{4}$, filtered and the solvent was evaporated under reduced pressure to give 5 .

Yield: $0.28 \mathrm{~g}$ (73\%); white solid; $\mathrm{mp} 120-123^{\circ} \mathrm{C}$.

IR (neat): 3355, 2900, 1730, 1135, 1015, 780, $553 \mathrm{~cm}^{-1}$.

${ }^{1} \mathrm{H}$ NMR $\left(400 \mathrm{MHz}, \mathrm{CDCl}_{3}\right): \delta=11.32(\mathrm{~s}, 1 \mathrm{H}), 9.67(\mathrm{~s}, 1 \mathrm{H}), 6.89(\mathrm{~s}$, $1 \mathrm{H}), 6.53(\mathrm{~s}, 1 \mathrm{H}), 6.41(\mathrm{~s}, 1 \mathrm{H}), 3.92(\mathrm{~s}, 3 \mathrm{H})$. 
${ }^{13} \mathrm{C}$ NMR $\left(100 \mathrm{MHz}, \mathrm{CDCl}_{3}\right): \delta=193.7,159.6,154.3,140.6,112.9$, 108.9, 103.2, 56.5 .

HRMS: $m / z[\mathrm{M}-\mathrm{H}]^{+}$calcd for $\mathrm{C}_{8} \mathrm{H}_{7} \mathrm{O}_{4}$ : 167.1123; found: 167.1129 .

\section{7-Hydroxy-6-methoxy-2H-chromen-2-one [Scopoletin (6)]}

To a stirred solution of $\mathbf{5}(0.2 \mathrm{~g}, 1.11 \mathrm{mmol})$ in $N, N$-diethylaniline (10 $\mathrm{mL}$ ) was added ethyl (triphenylphosporanylidene) acetate $(0.49 \mathrm{~g}$, $1.42 \mathrm{mmol}$ ) and the resulting mixture was heated to reflux for $12 \mathrm{~h}$. After completion of the reaction (monitored by TLC), the solvent was removed under reduced pressure. The resulting brown oily residue was purified by column chromatography, eluting with EtOAc-hexane (4:6) mixture to afford 6.

Yield: $0.16 \mathrm{~g}$ (70\%); yellow solid; $\mathrm{mp} 202-205^{\circ} \mathrm{C}$.

IR (neat): 3365, 2875, 1710, 1562, 1120, 1070, 828, $580 \mathrm{~cm}^{-1}$.

${ }^{1} \mathrm{H}$ NMR (400 MHz, $\mathrm{CDCl}_{3}$ ): $\delta=7.60(\mathrm{~d}, J=7.6 \mathrm{~Hz}, 1 \mathrm{H}), 6.92(\mathrm{~s}, 1 \mathrm{H})$, $6.85(\mathrm{~s}, 1 \mathrm{H}), 6.27$ (d, J = 7.6 Hz, $1 \mathrm{H}), 6.18(\mathrm{~s}, 1 \mathrm{H}), 3.96(\mathrm{~s}, 3 \mathrm{H})$.

${ }^{13} \mathrm{C}$ NMR $\left(100 \mathrm{MHz}, \mathrm{CDCl}_{3}\right): \delta=161.5,150.2,149.7,143.9,143.3$, 113.4, 111.5, 107.5, 103.2, 56.4.

HRMS: $m / z[\mathrm{M}+\mathrm{H}]^{+}$calcd for $\mathrm{C}_{10} \mathrm{H}_{9} \mathrm{O}_{4}$ : 193.0490; found: 193.0495 .

\section{(E)-7-[(3,7-Dimethylocta-2,6-dien-1-yl)oxy]-6-methoxy-2H- chromen-2-one (7)}

To a stirred solution of geranyl bromide ( $84 \mathrm{mg}, 0.39 \mathrm{mmol}$ ) in DMF ( 5 $\mathrm{mL}$ ) was added $\mathrm{K}_{2} \mathrm{CO}_{3}(71 \mathrm{mg}, 0.52 \mathrm{mmol}), \mathrm{KI}$ (cat) and a solution of scopoletin 6 ( $50 \mathrm{mg}, 0.26 \mathrm{mmol}$ ) in $\operatorname{DMF}(5 \mathrm{~mL})$. The resulting mixture was stirred for $2 \mathrm{~h}$ at r.t. After completion of reaction (monitored by TLC) the mixture was diluted with EtOAc $(10 \mathrm{~mL})$, quenched with cold water and further extracted with EtOAc $(2 \times 10 \mathrm{~mL})$. The combined organic layers were washed with brine, dried over $\mathrm{Na}_{2} \mathrm{SO}_{4}$, filtered and concentrated under reduced pressure. The crude product was purified by column chromatography, eluting with EtOAc-hexane (3:7) mixture to give 7 .

Yield: $70 \mathrm{mg}$ (82\%); amorphous solid.

IR (neat): 3022, 2875, 1735, 1245, 915, $750 \mathrm{~cm}^{-1}$.

${ }^{1} \mathrm{H}$ NMR (400 MHz, $\mathrm{CDCl}_{3}$ ): $\delta=7.61(\mathrm{~d}, J=9.5 \mathrm{~Hz}, 1 \mathrm{H}), 6.85(\mathrm{~s}, 1 \mathrm{H})$, $6.83(\mathrm{~s}, 1 \mathrm{H}), 6.27(\mathrm{~d}, J=9.5 \mathrm{~Hz}, 1 \mathrm{H}), 5.48(\mathrm{td}, J=6.4,1.2 \mathrm{~Hz}, 1 \mathrm{H}), 5.07$ (tt, $J=6.7,2.7 \mathrm{~Hz}, 1 \mathrm{H}), 4.70(\mathrm{~d}, J=6.4 \mathrm{~Hz}, 2 \mathrm{H}), 3.91(\mathrm{~s}, 3 \mathrm{H}), 2.15-$ $1.96(\mathrm{~m}, 4 \mathrm{H}), 1.77(\mathrm{~s}, 3 \mathrm{H}), 1.65(\mathrm{~s}, 3 \mathrm{H}), 1.59(\mathrm{~s}, 3 \mathrm{H})$.

${ }^{13} \mathrm{C}$ NMR $\left(100 \mathrm{MHz}, \mathrm{CDCl}_{3}\right): \delta=161.5,152.1,149.9,146.6,143.3$, $142.1,131.9,123.6,118.4,113.3,111.3,108.0,101.2,66.3,56.4,39.5$, 26.2, 25.6, 17.7, 16.8 .

HRMS: $m / z[\mathrm{M}+\mathrm{H}]^{+}$calcd for $\mathrm{C}_{20} \mathrm{H}_{25} \mathrm{O}_{4}$ : 329.1737; found: 329.1747 .

7-\{[(2E,6E)-8-Hydroxy-3,7-dimethylocta-2,6-dien-1-yl $]$ oxy $\}-6-m e-$ thoxy-2H-chromen-2-one [Artekeiskeanol A (1)]

To a stirred solution of $\mathrm{SeO}_{2}(67 \mathrm{mg}, 0.60 \mathrm{mmol})$ in TBHP $(4 \mathrm{~mL})$ and $\mathrm{CH}_{2} \mathrm{Cl}_{2}(1 \mathrm{~mL})$, cooled to $0{ }^{\circ} \mathrm{C}$, was added a solution of 7 (50 mg, 0.15 $\mathrm{mmol})$ in $\mathrm{CH}_{2} \mathrm{Cl}_{2}(1 \mathrm{~mL})$. The resulting mixture was then allowed to warm to r.t. and stirred for $4 \mathrm{~h}$. After completion of reaction (confirmed by TLC), the reaction was quenching with saturated aqueous sodium thiosulfate and extracted with ether $(2 \times 10 \mathrm{~mL})$. The combined organic layers were washed with brine, dried over $\mathrm{Na}_{2} \mathrm{SO}_{4}$, filtered and evaporated under reduced pressure to give $\mathbf{1}$.

Yield: $30 \mathrm{mg}$ (60\%); low-melting solid.

IR (neat): 3345, 3040, 2956, 1421, 1616, 1276, 1145, $758 \mathrm{~cm}^{-1}$.
${ }^{1} \mathrm{H}$ NMR (400 MHz, $\mathrm{CDCl}_{3}$ ): $\delta=7.61(\mathrm{~d}, J=9.5 \mathrm{~Hz}, 1 \mathrm{H}), 6.85(\mathrm{~s}, 1 \mathrm{H}$ ), $6.83(\mathrm{~s}, 1 \mathrm{H}), 6.28(\mathrm{~d}, J=9.5 \mathrm{~Hz}, 1 \mathrm{H}), 5.48(\mathrm{tdd}, J=6.4,2.3,1.2 \mathrm{~Hz}, 1 \mathrm{H})$, 5.37 (tdd, $J=6.9,2.6,1.4 \mathrm{~Hz}, 1 \mathrm{H}), 4.69(\mathrm{~d}, J=6.4 \mathrm{~Hz}, 2 \mathrm{H}), 3.97(\mathrm{~s}, 2 \mathrm{H})$, $3.91(\mathrm{~s}, 3 \mathrm{H}), 2.20(\mathrm{~m}, 2 \mathrm{H}), 2.10(\mathrm{~m}, 2 \mathrm{H}), 1.78(\mathrm{~s}, 3 \mathrm{H}), 1.66(\mathrm{~s}, 3 \mathrm{H})$.

${ }^{13} \mathrm{C}$ NMR $\left(100 \mathrm{MHz}, \mathrm{CDCl}_{3}\right): \delta=161.5,152.1,149.9,146.6,143.3$, $141.5,135.4,125.0,118.8,113.3,111.3,108.0,101.2,68.8,66.3,56.3$, 39.0, 25.5, 16.8, 13.7.

HRMS: $m / z$ [M - H] calcd for $\mathrm{C}_{20} \mathrm{H}_{23} \mathrm{O}_{5}$ : 343.1540; found: 343.1551 .

\section{3,5-Dibromo-2,4-dihydroxybenzaldehyde (8)}

To a stirred solution of 2,4-dihydroxybenzaldehyde ( $0.50 \mathrm{~g}, 3.6 \mathrm{mmol})$ in EtOH $(10 \mathrm{~mL})$ was added bromine $(0.22 \mathrm{~mL}, 9.1 \mathrm{mmol})$ dropwise at r.t. and the mixture was stirred for $1 \mathrm{~h}$. After completion of the reaction (monitored by TLC), the reaction was quenched with saturated aqueous sodium thiosulfate and extracted with EtOAc $(2 \times 15 \mathrm{~mL})$. The combined organic extracts were washed with brine, dried over $\mathrm{Na}_{2} \mathrm{SO}_{4}$, filtered, and the solvent was evaporated under reduced pressure to give 8 .

Yield: $1.00 \mathrm{~g}$ (98\%); white solid; $\mathrm{mp} 197-200{ }^{\circ} \mathrm{C}$.

IR (neat): 3263, 3052, 2923, 1725, 1467, 785, $555 \mathrm{~cm}^{-1}$.

$\left.{ }^{1} \mathrm{H} \mathrm{NMR} \mathrm{(400} \mathrm{MHz,} \mathrm{CDCl}_{3}\right): \delta=11.98(\mathrm{~s}, 1 \mathrm{H}), 9.68(\mathrm{~s}, 1 \mathrm{H}), 7.70(\mathrm{~s}$, $1 \mathrm{H}), 6.64(\mathrm{~s}, 1 \mathrm{H})$.

${ }^{13} \mathrm{C}$ NMR $\left(100 \mathrm{MHz}, \mathrm{CDCl}_{3}\right): \delta=193.4,159.6,156.2,136.0,116.2$, 100.0, 98.9.

HRMS: $m / z$ [M - H] calcd for $\mathrm{C}_{7} \mathrm{H}_{3} \mathrm{Br}_{2} \mathrm{O}_{3}$ : 292.7542; found: 292.7551.

\section{2,4-Dihydroxy-3,5-dimethoxybenzaldehyde (9)}

To stirred solution of sodium methoxide $(1.64 \mathrm{~g}, 30.5 \mathrm{mmol})$ in DMF $(20 \mathrm{~mL})$ was added dibromo compound $8(0.9 \mathrm{~g}, 3.05 \mathrm{mmol})$ and $\mathrm{CuCl}$ ( $30 \mathrm{mg}, 0.3 \mathrm{mmol}$ ), and the reaction mixture was heated to reflux for $8 \mathrm{~h}$, with vigorous stirring. After completion of the reaction (monitored by TLC), the solvent was removed under vacuum, ice was added, and the mixture was stirred for $20 \mathrm{~min}$. The mixture was then neutralized with $\mathrm{HCl}$, stirred for another $20 \mathrm{~min}$ and extracted with $\mathrm{CHCl}_{3}(2 \times 15 \mathrm{~mL})$. The combined organic layers were washed with brine, dried over $\mathrm{Na}_{2} \mathrm{SO}_{4}$, filtered, and the solvent was evaporated under reduced pressure to give $\mathbf{9}$.

Yield: $0.45 \mathrm{~g}$ (75\%); white solid; $\mathrm{mp} 87-89^{\circ} \mathrm{C}$.

IR (neat): 3379, 2924, 1735, 1645, 1324, 1137, 1088, 745, $553 \mathrm{~cm}^{-1}$.

$\left.{ }^{1} \mathrm{H} \mathrm{NMR} \mathrm{(400} \mathrm{MHz,} \mathrm{CDCl}_{3}\right): \delta=11.31(\mathrm{~s}, 1 \mathrm{H}), 9.7(\mathrm{~s}, 1 \mathrm{H}), 6.75(\mathrm{~s}, 1 \mathrm{H})$, 6.42 (brs, $1 \mathrm{H}), 4.01(\mathrm{~s}, 3 \mathrm{H}), 3.91(\mathrm{~s}, 3 \mathrm{H})$.

${ }^{13} \mathrm{C}$ NMR $\left(100 \mathrm{MHz}, \mathrm{CDCl}_{3}\right): \delta=194.5,151.6,146.9,141.2,134.5$, 112.9, 108.9, 60.9, 56.6.

HRMS: $m / z$ [M - H] ${ }^{+}$calcd for $\mathrm{C}_{9} \mathrm{H}_{9} \mathrm{O}_{5}$ : 197.0440; found: 197.0445 .

7-Hydroxy-6,8-dimethoxy-2H-chromen-2-one [isofraxidin (10)] The above procedure for $\mathbf{6}$ was followed for the preparation of $\mathbf{1 0}(0.3$ g, $0.30 \mathrm{mmol})$.

Yield: $0.24 \mathrm{~g}(72 \%) ; \mathrm{mp} 146-148{ }^{\circ} \mathrm{C}$.

IR (neat): 3352, 2924, 1702, 1459, 1310, 1118, 1080, $590 \mathrm{~cm}^{-1}$.

${ }^{1} \mathrm{H}$ NMR (400 MHz, $\left.\mathrm{CDCl}_{3}\right): \delta=7.60(\mathrm{~d}, J=9.5 \mathrm{~Hz}, 1 \mathrm{H}), 6.66(\mathrm{~s}, 1 \mathrm{H})$, $6.28(\mathrm{~d}, J=9.5 \mathrm{~Hz}, 1 \mathrm{H}), 6.22(\mathrm{~s}, 1 \mathrm{H}), 4.09$ (s, $3 \mathrm{H}), 3.94(\mathrm{~s}, 1 \mathrm{H})$.

${ }^{13} \mathrm{C}$ NMR $\left(100 \mathrm{MHz}, \mathrm{CDCl}_{3}\right): \delta=160.6,144.6,143.8,143.0,142.5$, 134.4, 113.4, 111.2, 103.2, 61.6, 56.5.

HRMS: $m / z[\mathrm{M}+\mathrm{H}]^{+}$calcd for $\mathrm{C}_{11} \mathrm{H}_{11} \mathrm{O}_{5}$ : 223.1376; found: 223.1370 . 
(E)-7-[(3,7-Dimethylocta-2,6-dien-1-yl)oxy]-6,8-dimethoxy-2Hchromen-2-one [altissamacoumarin D (3)]

To a stirred solution of geranyl bromide ( $73 \mathrm{mg}, 0.33 \mathrm{mmol}$ ) in DMF ( 5 $\mathrm{mL}$ ) was added $\mathrm{K}_{2} \mathrm{CO}_{3}(62 \mathrm{mg}, 0.45 \mathrm{mmol})$, $\mathrm{KI}$ (cat) and a solution of isofraxidin (10; $50 \mathrm{mg}, 0.23 \mathrm{mmol}$ ) in DMF ( $5 \mathrm{~mL}$ ). The resulting mixture was stirred for $2 \mathrm{~h}$ at r.t.. After completion of reaction was confirmed by TLC, the mixture was diluted with EtOAc $(10 \mathrm{~mL})$ and quenched with cold water and extracted with EtOAc $(2 \times 10 \mathrm{~mL})$. The combined organic phases were washed with brine, dried over $\mathrm{Na}_{2} \mathrm{SO}_{4}$, filtered and concentrated under reduced pressure. The crude product was purified by column chromatography, eluting with EtOAc-hexane (3:7) mixture to give 7.

Yield: $64 \mathrm{mg}$ (80\%); amorphous solid.

IR (neat): 3022, 2875, 1435, 1245, 910, $749 \mathrm{~cm}^{-1}$.

${ }^{1} \mathrm{H}$ NMR $\left(400 \mathrm{MHz}, \mathrm{CDCl}_{3}\right): \delta=7.61(\mathrm{~d}, J=9.4 \mathrm{~Hz}, 1 \mathrm{H}), 6.66(\mathrm{~s}, 1 \mathrm{H})$, $6.33(\mathrm{~d}, J=9.5 \mathrm{~Hz}, 1 \mathrm{H}), 5.56(\mathrm{td}, J=7.2,1.2 \mathrm{~Hz}, 1 \mathrm{H}), 5.07(\mathrm{~m}, 1 \mathrm{H})$, 4.68 (d, J = 7.2 Hz, $2 \mathrm{H}), 4.03(\mathrm{~s}, 3 \mathrm{H}), 3.89$ (s, $3 \mathrm{H}), 2.06$ (m, $4 \mathrm{H}), 1.70$ (s, $3 \mathrm{H}), 1.66(\mathrm{~s}, 3 \mathrm{H}), 1.59(\mathrm{~s}, 3 \mathrm{H})$.

${ }^{13} \mathrm{C}$ NMR $\left(100 \mathrm{MHz}, \mathrm{CDCl}_{3}\right): \delta=160.5,150.6,144.8,143.5,142.9$, $142.5,141.7,131.7,123.8,119.5,115.1,114.4,103.5,70.2,61.7,56.2$, 39.6, 26.3, 25.6, 17.6, 16.3 .

HRMS: $m / z[M+H]^{+}$calcd for $\mathrm{C}_{21} \mathrm{H}_{27} \mathrm{O}_{5}$ : 359.1737; found: 359.1747 .

7-\{[(2E,6E)-8-Hydroxy-3,7-dimethylocta-2,6-dien-1-yl]oxy\}-6,8dimethoxy-2H-chromen-2-one [Artekeiskeanol C (2)]

The above procedure was followed for the preparation of $\mathbf{2}(50 \mathrm{mg}$, $0.14 \mathrm{mmol})$. Yield: $33 \mathrm{mg}$ (63\%).

IR (neat): 3345, 3054, 2986, 1721, 1616, 1275, 1148, $758 \mathrm{~cm}^{-1}$.

${ }^{1} \mathrm{H}$ NMR (400 MHz, $\left.\mathrm{CDCl}_{3}\right): \delta=7.61(\mathrm{~d}, J=9.5 \mathrm{~Hz}, 1 \mathrm{H}), 6.66(\mathrm{~s}, 1 \mathrm{H})$, $6.34(\mathrm{~d}, J=9.4 \mathrm{~Hz}, 1 \mathrm{H}), 5.56(\mathrm{tdd}, J=7.2,2.3,1.2 \mathrm{~Hz}, 1 \mathrm{H}), 5.37(\mathrm{t}$, $J=6.6, \mathrm{~Hz}, 1 \mathrm{H}), 4.67$ (d, J = 7.2 Hz, $2 \mathrm{H}), 4.03(\mathrm{~s}, 3 \mathrm{H}), 3.98(\mathrm{~s}, 3 \mathrm{H}), 3.89$ (s, $3 \mathrm{H}), 2.15-2.20(\mathrm{~m}, 2 \mathrm{H}), 2.02-2.10(\mathrm{~m}, 2 \mathrm{H}), 1.70(\mathrm{~s}, 3 \mathrm{H}), 1.66$ (s, $3 \mathrm{H})$.

${ }^{13} \mathrm{C}$ NMR $\left(100 \mathrm{MHz}, \mathrm{CDCl}_{3}\right): \delta=160.5,150.6,144.8,143.4,142.9$, 142.1, 141.8, 135.2, 125.3, 119.9, 115.1, 114.4, 103.6, 70.2, 68.8, 61.7, 56.3, 39.1, 25.7, 16.3, 13.7 .

HRMS: $m / z[\mathrm{M}+\mathrm{H}]^{+}$calcd for $\mathrm{C}_{21} \mathrm{H}_{26} \mathrm{O}_{6} \mathrm{Na}$ : 397.1612; found: 397.1621 .

\section{Funding Information}

A.T. and K.B. are grateful to UGC-New Delhi and CSIR-New Delhi, respectively, for providing Fellowships.

\section{Acknowledgment}

A.T. and K.B. are grateful to the Director CSIR-IICT for providing facilities.

\section{Supporting Information}

Supporting information for this article is available online at https://doi.org/10.1055/s-0037-1610873.

\section{References}

(1) (a) Estevez-Braun, A.; Gonzalez, A. G. Nat. Prod. Rep. 1997, 14, 465. (b) Newman, D. J.; Cragg, G. M. J. Nat. Prod. 2007, 70, 461. (c) Lachance, H.; Wetzel, S.; Kumar, K.; Waldmann, H. J. Med. Chem. 2012, 55, 5989.

(2) (a) Cai, X.; Yang, J.; Zhou, J.; Lu, W.; Hu, C.; Gu, Z.; Huo, J.; Wang, X.; Cao, P. Bioorg. Med. Chem. 2013, 21, 84. (b) Li, L.; Zhao, P.; Hu, J.; Liu, J.; Liu, Y.; Wang, Z.; Xia, Y.; Dai, Y.; Chen, L. Eur. J. Med. Chem. 2015, 93, 300.

(3) (a) Yamazaki, T.; Tokiwa, T. Biol. Pharm. Bull. 2010, 3, 1716. (b) Li, J.; Li, X.; Li, Z.; Zhang, L.; Liu, Y.; Ding, H.; Yin, S. Food Funct. 2017, 8, 2886.

(4) (a) Dao, T. T.; Tran, T. L.; Kim, J.; Nguyen, P. H.; Lee, E. H.; Park, J.; Jang, I. S.; Oh, W. K. J. Nat. Prod. 2012, 75, 1332. (b) Hwang, S. W.; Lee, J. R.; Lee, J.; Kwon, H. S.; Yang, M. S.; Park, K. H. Heterocycles 2005, 65, 1963.

(5) (a) Kwak, J. H.; Jang, W. Y.; Zee, O. P.; Lee, K. R. Planta Med. 1997, $63,474$.

(6) Kwak, J. H.; Lee, K. B.; Schmitz, F. J. J. Nat. Prod. 2001, 64, 1081.

(7) Honga, J.; Sasaki, H.; Hirasawa, N.; Ishihara, K.; Kwak, J. H.; Zee, O.; Schmiz, F. J.; Seyama, T.; Ohuchi, K. Planta Med. 2009, 75, 1494.

(8) (a) Narala, S. G.; Nagalatha, G.; Narsaiah, A. V. ARKIVOC 2018, (vii), 495. (b) Nagalatha, G.; Ganesh, N. S.; Narsaiah, A. V. SynOpen 2018, 2, 251. (c) Ghogare, R. S.; Wadavrao, S. B.; Narsaiah, A. V. Helv. Chim. Acta 2016, 99, 247. (d) Baikadi, K.; Talakokkula, A.; Narsaiah, A. V. SynOpen 2019, 3, 26. (e) Narala, S. G.; Nagalatha, G.; Narsaiah, A. V. Nat. Prod. Res. 2019, 1577843.

(9) (a) Manchand, P. S.; Belica, P. S.; Wong, H. S. Synth. Commun. 1990, 20, 2659. (b) Wanmu, C. Indian J. Chem., Sect. B: Org. Chem. Incl. Med. Chem. 1996, 35, 1085.

(10) (a) Aalten, H. L.; Koten, G. V.; Grove, D. M.; Kuilman, T.; Piekstra, O. G.; Hulshof, L. A.; Sheldon, R. A. Tetrahedron 1989, 45, 5565. (b) Rouessac, F.; Leclerc, A. Synth. Commun. 1993, 23, 1147.

(11) (a) Harayama, T. J. Pharm. Soc. Jpn. 2006, 126, 543. (b) Wenqing, G.; Qingyong, L.; Jian, C.; Zhichao, W.; Changlong, H. Molecules 2013, 18, 15613. (c) Jain, P. K.; Himanshu, J. J. Appl. Pharm. Sci. 2012, 2, 236.

(12) Geranyl bromide (11) was prepared as per reported procedure, see: Hanessian, S.; Cooke, N. G.; DeHoff, B.; Sakito, Y. J. Am. Chem. Soc. 1990, 112, 5276.

(13) (a) Silva, A. C.; Benelkebir, H.; Lopes, R. S. C.; Lopes, C. C.; Ganesan, A. J. Braz. Chem. Soc. 2018, 29, 1157. (b) Allen, C. F. H.; Gates, J. W. Jr. Org. Synth. Coll. Vol. 3 1955, 3, 140. (c) Zep, A.; Wojicik, M. W.; Lewandowski, W.; Sitkowska, K.; Prominski, J. M.; Pociecha, D.; Gorecka, E. Angew. Chem. Int. Ed. 2014, 53, 13725.

(14) (a) Riley, H. L.; Morley, J. F.; Friend, N. A. C. J. Chem. Soc. 1932, 1875. (b) Marshall, J. A.; Lebreton, J.J. Org. Chem. 1988, 53, 4108. 